\title{
Globalizing Sport Studies series editor's preface
}

There is now a considerable amount of expertise nationally and internationally in the social scientific and cultural analysis of sport in relation to the economy and society more generally. Contemporary research topics, such as sport and social justice, science and technology and sport, global social movements and sport, sports mega-events, sports participation and engagement, and the role of sport in social development, suggest that sport and social relations need to be understood in non-Western developing economies, as well as European, North American and other advanced capitalist societies. The current high global visibility of sport makes this an excellent time to launch a major new book series that takes sport seriously, and makes this research accessible to a wide readership.

The series Globalizing Sport Studies is thus in line with a massive growth of academic expertise, research output and public interest in sport worldwide. At the same time, it seeks to use the latest developments in technology and the economics of publishing to reflect the most innovative research into sport in society currently underway in the world. The series is multidisciplinary, although primarily based on the social-sciences and cultural-studies approaches to sport.

The broad aims of the series are to: act as a knowledge hub for social scientific and cultural studies research in sport, including, but not exclusively, anthropological, economic, geographical, historical, political science and sociological studies; contribute to the expanding field of research on sport in society in the United Kingdom and internationally by focusing on sport at regional, national and international levels; create a series for both senior and more junior researchers that will become synonymous with cutting-edge research, scholarly opportunities and academic development; promote innovative discipline-based, multi-, inter- and trans-disciplinary theoretical and methodological approaches to researching sport in society; provide an English-language outlet for high quality non-English writing on sport in society; publish broad overviews, original empirical research studies and classic studies from non-English sources; and 
thus attempt to realise the potential for globalizing sport studies through open-content licensing with 'Creative Commons'.

Localizing Global Sport for Development is the result of extensive research by the authors into 'sport for development' (SfD) in Zambia since 2006. Whilst being informed by the burgeoning SfD literature, the authors add to this in two distinctive ways: detailed examination of the interaction between the global and local dimensions of SfD policies and practices over a long period of time in a particular location, and critical reflections on the research methods that have underpinned their work and that of others in SfD to date. In so doing, the authors explore the implications for future work in the field of focusing on 'the local'.

The first three substantive chapters of the book set the scene. They locate the authors' research within the SfD literature, examine the suitability of Zambia as a context for a 'localized' approach to $\mathrm{SfD}$ research, and assess $\mathrm{SfD}$ policies and practices in general. Aligned with empirically orientated development-studies literature, a distinctive feature of the book is the self-reflection the four authors bring to bear on the process of knowledge production as they have worked together in various combinations to conduct their research. Since 2006 they have conducted ten research projects addressing several development issues, such as HIV/AIDS and gender inequality, that were pertinent to Zambia and have been addressed by SfD programmes elsewhere. The book thus provides a more wideranging, detailed and holistic account of SfD than many previous studies.

The following three chapters focus on the actors, especially young people, involved in $\mathrm{SfD}$ and the local context within which $\mathrm{SfD}$ operates in Zambia. A distinctive feature of the discussion considers the way that young people are often well equipped to provide critical and knowledgeable accounts of the issues they face and which SfD organizations seek to alleviate. These chapters also examine young people's engagement in, and responses to, various dimensions of SfD provision.

Unlike other accounts of SfD, Localizing Global Sport for Development provides a richly detailed examination of the varied practices and experiences of Zambian $\mathrm{SfD}$ and explores the contribution of locally grounded research to understanding $\mathrm{SfD}$ in general. In doing so, the relationships between the local dimensions of SfD and broader, global, influences are assessed. The book suggests that similar in-depth, contextually grounded, and localized, understandings will be central to improving $\mathrm{SfD}$ practices and policies in the future. 